\title{
NUMERICAL SOLUTION OF MIXED LINEAR VOLTERRA- FREDHOLM INTEGRAL EQUATIONS BY MODIFIED BLOCK PULSE FUNCTIONS
}

\author{
AYYUBi AHMAD \\ Department of Mathematics, Faculty of Applied Science and Technology, Ahmad Dahlan University, \\ Yogyakarta, Indonesia \\ ayyubiahmad1@gmail.com
}

\begin{abstract}
A numerical method based on modified block pulse functions is proposed for solving the mixed linear Volterra-Fredholm integral equations. An integration operational matrix of modified block pulse functions is obtained on interval $[0, T)$. A modified block pulse functions and their operational matrix of integration, the mixed linear Volterra-Fredholm integral equations can be reduced to a linear system of algebraic equations. The rate of convergence is $\mathcal{O}(h)$ and error analysis of the proposed method are discussed. Some examples are provided to show that the proposed method have a good degree of accuracy.
\end{abstract}

Keywords: Integration Operational Matrix, Itô Integral, Mixed Linear Volterra-Fredholm Integral Equations, $\varepsilon$ MBPFs

\section{Introduction}

An integral equation is an equation in which an unknown function appears under one or more integration signs. The integral equations are found in various applied areas including mechanics, physics, and engineering. The subject of integral equations are used as mathematical models for many and varied physical situations. It also arise as representation formulas for the solutions of differential equations.

The mixed linear Volterra-Fredholm integral equation is a combination of disjoint linear Volterra and Fredholm integrals that appear in an integral equation. The mixed linear Volterra-Fredholm integral equations arise from parabolic boundary value problems, mathematical modelling of the spatio-temporal development of an epidemic, various physical, biological, and chemical applications $[9,10]$. There are several techniques for approximating the solution such as designing neural network [1], linear programming problem [5], Taylor expansion method [2], homotopy perturbation method [4], Legendre collocation method [7], and Bernstein polynomials method [8].

In this paper, the modified block pulse functions is applied for solving the following mixed linear Volterra-Fredholm integral equations

$$
X(t)=f(t)+\int_{0}^{t} \int_{\alpha}^{\beta} K(x, r) X(r) d r d x,
$$

where $X(t)$ is the unknown function, $f(t)$ is analytic function and $K(x, r)$ is the kernels of $L^{2}$ functions. 


\section{Methodology}

A set of $\varepsilon$ modified block pulse functions $\psi_{i}(t), i=0,1, \ldots, m$ on the interval $[0, T)$ are defined as

$$
\begin{gathered}
\psi_{0}(t)= \begin{cases}1 & t \in[0, h-\varepsilon)=I_{0}, \\
0 & \text { otherwise, }\end{cases} \\
\psi_{i}(t)= \begin{cases}1 & t \in[i h-\varepsilon,(i+1) h-\varepsilon)=I_{i}, \\
0 & \text { otherwise, }\end{cases}
\end{gathered}
$$

for $i=1,2, \ldots, m-1$, and

$$
\psi_{m}(t)= \begin{cases}1 & t \in[T-\varepsilon, T)=I_{m} \\ 0 & \text { otherwise }\end{cases}
$$

with a positive integer value for $m$ and $h=\frac{T}{m}$.

Similar to BPFs, the important properties of $\varepsilon$ MBPFs are as follows

- Disjointness:

where $i, j=0, \ldots, m$.

$$
\psi_{i}(t) \psi_{j}(t)= \begin{cases}\psi_{i}(t) & i=j \\ 0 & i \neq j\end{cases}
$$

- Orthogonality:

$$
\int_{0}^{T} \psi_{i}(t) \psi_{j}(t) d t=h \delta_{i j}
$$

where $i, j=1, \ldots, m-1$ and $\delta_{i j}$ is Kronecker delta.

- Completeness:

where

$$
\int_{0}^{T} f^{2}(t) d t=\sum_{i=0}^{\infty} f_{i}^{2}\left\|\psi_{i}(t)\right\|^{2},
$$

and $\Delta\left(I_{i}\right)$ is length of interval $I_{i}$.

$$
f_{i}=\frac{1}{\Delta\left(I_{i}\right)} \int_{0}^{T} f(t) \psi_{i}(t) d t
$$

Rewriting Eq. (2) in the vector form we have

in which

$$
f(t) \simeq \sum_{i=0}^{m} f_{i} \psi_{i}(t)=F^{T} \Psi(t)=\Psi^{T}(t) F,
$$

$$
F=\left(\begin{array}{llll}
f_{0} & f_{1} & \ldots & f_{m}
\end{array}\right)^{T} \text { and } \Psi(t)=\left(\begin{array}{llll}
\psi_{0}(t) & \psi_{1}(t) & \ldots & \psi_{m}(t)
\end{array}\right)^{T} .
$$

Moreover, any two dimensional function $k(s, t) \in L^{2}\left(\left[0, T_{1}\right) \times\left[0, T_{2}\right)\right)$ can be expanded with respect to $\varepsilon$ MBPFs such as

$$
k(s, t)=\Psi^{T}(s) K \Phi(t)=\Phi^{T}(t) K^{T} \Psi(s),
$$

where $\Psi(s)$ and $\Phi(t)$ are $m_{1}$ and $m_{2}$ dimensional $\varepsilon$ MBPFs vectors respectively, and $K=$ $\left(k_{i j}\right), i=0,1, \ldots, m_{1}, j=0,1, \ldots, m_{2}$ is the $m_{1} \times m_{2} \varepsilon$ modified block pulse coefficient matrix with

$$
k_{i j}=\frac{1}{\Delta\left(I_{i}\right) \Delta\left(I_{j}\right)} \int_{0}^{T_{1}} \int_{0}^{T_{2}} k(s, t) \Psi_{i}(s) \Phi_{j}(t) d t d s,
$$

For convenience, we put $m_{1}=m_{2}=m$.

With defining $\Psi_{m+1}(t)=\left(\begin{array}{lllll}\psi_{0}(t) & \psi_{1}(t) & \ldots & \psi_{m}(t)\end{array}\right)^{T}$, we have 


$$
\Psi_{m+1}(t) \Psi_{m+1}^{T}(t)=\left(\begin{array}{cccc}
\psi_{0}(t) & 0 & \cdots & 0 \\
0 & \psi_{1}(t) & \cdots & 0 \\
\vdots & \vdots & \ddots & \vdots \\
0 & 0 & \cdots & \psi_{m}(t)
\end{array}\right)_{(m+1) \times(m+1)}
$$

Furthermore,

and

$$
\Psi_{m+1}^{T}(t) \Psi_{m+1}(t)=1
$$

$$
\Psi_{m+1}(t) \Psi_{m+1}^{T}(t) F=D_{F} \Psi_{m+1}(t)
$$

where $D_{F}$ usually denotes a diagonal matrix whose diagonal entries are related to a constant vector $F=\left(\begin{array}{llll}f_{0} & f_{1} & \ldots & f_{m}\end{array}\right)^{T}$.

Similar to BPFs,

$$
\int_{0}^{t} \Psi_{m+1}(s) d s \simeq Q \Psi_{m+1}(t),
$$

where the integration operational matrix $Q$ of $\varepsilon$ MBPFs is given by

$$
Q=\left(\begin{array}{cccc}
\frac{h-\varepsilon}{2} & h-\varepsilon & \cdots & h-\varepsilon \\
0 & \frac{h}{2} & \cdots & h \\
\vdots & \vdots & \ddots & \vdots \\
0 & 0 & \cdots & \frac{\varepsilon}{2}
\end{array}\right)_{(m+1) \times(m+1)}
$$

So, the integral of every function $f(t)$ can be approximated as follows

$$
\int_{0}^{t} f(s) d s \simeq \int_{0}^{t} F^{T} \Psi_{m+1}(s) d s \simeq F^{T} Q \Psi_{m+1}(t) .
$$

\section{Results}

\subsection{Solving Mixed Linear Volterra-Fredholm Integral Equations by Using $\varepsilon$ Modified Block Pulse Functions}

We consider following mixed linear Volterra-Fredholm integral equation

$$
X(t)=f(t)+\int_{0}^{t} \int_{\alpha}^{\beta} K(x, r) X(r) d r d x,
$$

We approximate functions $X(t), f(t)$, and $K(x, r)$ by $\varepsilon$ MBPFs:

$$
\begin{gathered}
X(t) \simeq W^{T} \Phi(t)=\Phi^{T}(t) W, \\
f(t) \simeq F^{T} \Phi(t)=\Phi^{T}(t) F, \\
K(x, r) \simeq \Phi^{T}(x) K \Phi(r)=\Phi^{T}(r) K^{T} \Phi(x) .
\end{gathered}
$$

In the above approximates, $W$ and $F$ are modified block pulse coefficients vector, and $K$ are modified block pulse coefficients matrix.

Substituting above approximation in Eq. (3), we get

$$
W^{T} \Phi(t) \simeq F^{T} \Phi(t)+\int_{0}^{t} \int_{\alpha}^{\beta} \Phi^{T}(x) K \Phi(r) \Phi^{T}(r) W d r d x
$$




$$
\begin{gathered}
W^{T} \Phi(t) \simeq F^{T} \Phi(t)+\int_{0}^{t} \Phi^{T}(x) K\left(\int_{\alpha}^{\beta} \Phi(r) \Phi^{T}(r) d r\right) W d x \\
W^{T} \Phi(t) \simeq F^{T} \Phi(t)+\int_{0}^{t} \Phi^{T}(x) K h I W d x \\
W^{T} \Phi(t) \simeq F^{T} \Phi(t)+\int_{0}^{t} \Phi^{T}(x) h K W d x \\
W^{T} \Phi(t) \simeq F^{T} \Phi(t)+W^{T}(h K)^{T} \int_{0}^{t} \Phi(x) d x \\
W^{T} \Phi(t) \simeq F^{T} \Phi(t)+W^{T}(h K)^{T} Q \Phi(t) \\
W^{T} \simeq F^{T}+W^{T}(h K)^{T} Q \\
W^{T}-W^{T}(h K)^{T} Q \simeq F^{T} \\
W^{T}\left(I-(h K)^{T} Q\right) \simeq F^{T}
\end{gathered}
$$

So, by setting $N=\left(I-(h K)^{T} Q\right)^{T}$ and replacing $\simeq$ by $=$, we have

$$
N W=F .
$$

Which is a linear system of equations with upper triangular coefficients matrix that gives the approximate modified block pulse coefficient of the unknown $X(t)$.

\subsection{Error Analysis}

In the following theorems, for simplicity we assume $T=1$ and $h=\frac{1}{m}$.

Theorem 3.2.1 If $\hat{f}_{m}(t)=\sum_{i=0}^{m} f_{i} \psi_{i}(t)$ and $f_{i}=\frac{1}{\Delta\left(I_{i}\right)} \int_{0}^{1} f(t) \psi_{i}(t) d t, i=0, \ldots, m$ then:

i. $\quad \delta=\int_{0}^{1}\left(f(t)-\sum_{i=0}^{m} f_{i} \psi_{i}(t)\right)^{2} d t$, achieves its minimum value.

ii. $\quad\left\{\hat{f}_{m}(t)\right\}$ approaches $f(t)$ pointwise.

iii. $\quad \int_{0}^{1} f^{2}(t) d t=\sum_{i=0}^{\infty} f_{i}^{2}\left\|\psi_{i}\right\|^{2}$

PROOF. Proof is like similar theorem in [3] but intervals of integration have to redefine as $I_{i}, i=0, \ldots, m$ in (3.1).

\section{Theorem 3.2.2 Assume:}

i. $f(t)$ is continuous and differentiable in $[-h, 1+h]$ with bounded derivative, that is $\left|f^{\prime}(t)\right|<M$.

ii. $\quad \hat{f}_{\frac{i h}{k}}(t), i=0, \ldots, k-1$, are correspondingly BPFs, $\frac{h}{k}$ MBPFs $\ldots, \frac{(k-1) h}{k}$ MBPFs expansions of $f(t)$ base on $m+1 \varepsilon$ MBPFs over interval $[0,1)$.

iii. $\quad \bar{f}(t)=\frac{1}{k} \sum_{i=0}^{k-1} \hat{f}_{\frac{i h}{k}}(t)$.

Then

$$
\left\|f(t)-\hat{f}_{\frac{i h}{k}}(t)\right\|=\mathcal{O}(h), \quad \text { and } \quad\|f(t)-\bar{f}(t)\|=\mathcal{O}\left(\frac{h}{k}\right) \text { in }[h, 1-h] .
$$

PROOF. Trapezoidal rule for integral is 


$$
\begin{aligned}
\int_{a}^{b} f(t) d t & =\frac{b-a}{2}(f(a)+f(b))-\frac{(b-a)^{3} f^{\prime \prime}(\eta)}{12} \\
& =\frac{b-a}{2}(f(a)+f(b))+E, \quad \eta \in[a, b],
\end{aligned}
$$

where $E$ is error of integration. Suppose $t_{i}=\frac{i}{m}=i h$ and $I_{i}=\left[t_{i-1}, t_{i}\right]$. The representation error when $f(t)$ is represented by a series of BPFs over every subinterval $\left[t_{i}, t_{i}+\frac{h}{k}\right], i=$ $0, \ldots, m-1$ is

where $f_{i}=\frac{1}{h} \int_{i h}^{(i+1) h} f(t) d t$. From (4),

$$
e_{i}(t)=f(t)-f_{i} \psi_{i}(t)=f(t)-f_{i}
$$

$$
f_{i}=\frac{1}{2}\left(f\left(t_{i}\right)+f\left(t_{i}+h\right)\right)+E .
$$

It is obvious that if $f(t)=C$ (constant), then $e_{i}(t)=0$. So, this error is computed for $f(t)=t$ in interval $\left[t_{i}, t_{i}+\frac{h}{k}\right], i=1, \ldots, m-1$.

For this function $E=0$, so

$$
e_{i}(t)_{\left[t_{i}, t_{i}+\frac{h}{k}\right]}=\left|t-f_{i}\right|=\left|t-\frac{t_{i}+t_{i+1}}{2}\right|=\left|t-\left(t_{i}+\frac{h}{2}\right)\right| \leq \frac{h}{2} .
$$

Then this error with BPFs is $\frac{h}{2} M$.

Similarly, the error when $f(t)$ is represented in a series of $\varepsilon$ MBPFs over every subinterval $\left[t_{i}, t_{i}+\frac{h}{k}\right]$ is

$$
\begin{aligned}
e_{i}(t)\left[t_{i}, t_{i}+\frac{h}{k}\right] & =\left|t-\left(\frac{\sum_{j=0}^{k-1}\left(t_{i}-\left(\frac{j h}{k}\right)+t_{i+1}-\left(\frac{j h}{k}\right)\right)}{2 k}\right)\right| \\
& =\left|t-\left(\frac{\sum_{j=0}^{k-1}\left(t_{i}-\left(\frac{j h}{k}\right)+t_{i}+h-\left(\frac{j h}{k}\right)\right)}{2 k}\right)\right| \\
& =\left|t-\left(t_{i}+\frac{h}{2}\right)-\frac{(k-1) h}{2 k}\right| \\
& \leq \frac{h}{2 k} .
\end{aligned}
$$

So, the error with $\varepsilon$ MBPFs is $\frac{h}{2 k} M$.

For $I_{0}$ in $\left[0, \frac{h}{k}\right]$ we have

So, the error is $\mathcal{O}\left(\frac{h}{4}\right)$ also for $I_{n}$.

$$
\begin{aligned}
e_{i}(t)_{\left[0, \frac{h}{k}\right]} & =\left|t-\sum_{j=0}^{k-1} \frac{h-\left(\frac{j h}{k}\right)}{2 k}\right| \\
& =\left|t-\left(\frac{h}{2}-\frac{(k-1) h}{4 k}\right)\right| \\
& =\left|t-\left(\frac{h}{4}+\frac{h}{4 k}\right)\right| \\
& =\mathcal{O}\left(\frac{h}{4}\right) .
\end{aligned}
$$


Now,

$$
\begin{aligned}
\left\|e_{i}(t)\right\|^{2} & =\int_{t_{i}}^{t_{i}+\frac{h}{k}}\left|e_{i}(t)\right|^{2} d t \\
& =\int_{t_{i}}^{t_{i}+\frac{h}{k}} \frac{h^{2}}{4 k^{2}} M^{2} d t \\
& =\frac{h^{3}}{4 k^{3}} M^{2}, \\
\|e\|^{2} & =\int_{0}^{1} e^{2}(t) d t \\
& =\int_{0}^{1}\left(\sum_{i=1}^{m} \sum_{j=0}^{k-1} e_{i}(t)\right)^{2} d t \\
& =\sum_{i=1}^{m} \sum_{j=0}^{k-1} \int_{0}^{1} e_{i}^{2}(t) d t \\
& =\sum_{i=1}^{m} \sum_{j=0}^{k-1}\left\|e_{i}(t)\right\|^{2} \\
& =\frac{1}{h} \cdot k \cdot \frac{h^{3}}{4 k^{3}} M^{2} \\
& =\frac{h^{2}}{4 k^{2}} M^{2} \cdot \\
&
\end{aligned}
$$

We define the representation error between $f(s, t)$ and its $2 \mathrm{D}-\varepsilon$ MBPFs expansion $f_{i j}$ over every subregion $D_{i j}$, is defined as

$$
e_{i j}(s, t)=f(s, t)-f_{i j},
$$

where $D_{i j}:=\left\{(s, t) \mid t_{i} \leq s \leq t_{i}+\frac{h}{k}, t_{j} \leq t \leq t_{j}+\frac{h}{k}\right\}$.

With Taylor's expansion and similarity to the above discussion,

$$
\|e(s, t)\|=\frac{h}{2 k} M .
$$

Theorem 3.2.3 Assume that

$$
P(\omega \in \Omega:\|u(\omega, t)\|<C)=1 .
$$

$\left\|k_{i}\right\|<C, i=1,2$.

Then

$$
\begin{aligned}
& \sup \left(E(\|(u-\bar{u})\|)^{2}\right)^{\frac{1}{2}}=\mathcal{O}\left(\frac{h}{k}\right), \quad t \in[h, 1-h] . \\
& 0 \leq t \leq T
\end{aligned}
$$

PROOF. For a complete proof see [6]. 


\subsection{Numerical Examples}

Example 3.3.1 Consider the following mixed linear Volterra-Fredholm integral equation,

where the exact solution is $f(t)=\cos t$.

$$
X(t)=\cos t-2 t+\int_{0}^{t} \int_{0}^{\pi}(s-r) X(r) d r d s,
$$

Table 1: The Exact and Approximation Solutions of Example 1

\begin{tabular}{|l|l|l|}
\hline \multirow{2}{*}{$\boldsymbol{t}$} & \multicolumn{2}{|c|}{$\boldsymbol{m}=\mathbf{1 0 2 5}$} \\
\cline { 2 - 3 } & Exact & Approximation \\
\hline 0 & 1 & 1 \\
\hline 0.6 & 0.8253 & 0.8224 \\
\hline 1.2 & 0.3626 & 0.3612 \\
\hline 1.8 & -0.2276 & -0.2272 \\
\hline 2.4 & -0.7372 & -0.5052 \\
\hline 3 & -0.99 & -0.9948 \\
\hline
\end{tabular}

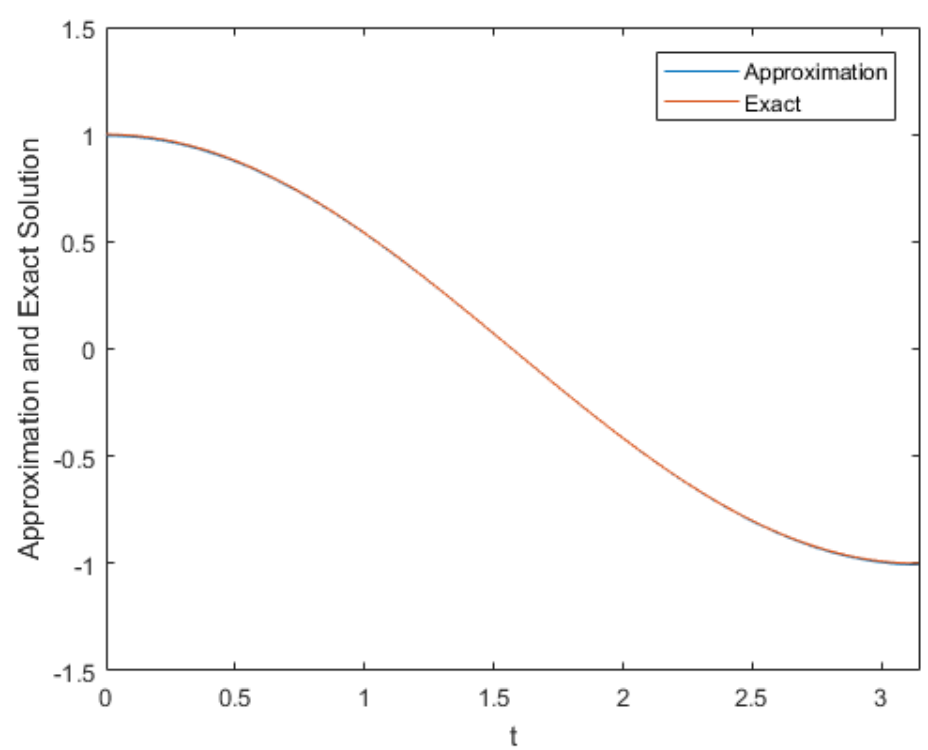

Figure 1: The Trajectory of The Exact and Approximation Solution of Example 1

Based on the graph above, it can be seen that the approximate and exact results coincide with each other at the interval $[0, \pi)$. At the beginning and end that if the number of partitions we take is getting bigger and $\varepsilon$ getting smaller, it will produce an approximation result that is closer to the exact.

Example 3.3.2 Consider the following mixed linear Volterra-Fredholm integral equation,

$$
X(t)=\cos t+\sin t-t^{2}+\frac{\pi}{2} t+\int_{0}^{t} \int_{0}^{\frac{\pi}{2}}(s-r) X(r) d r d s,
$$

where the exact solution is $f(t)=\cos t$. 
Table 2: The Exact and Approximation Solutions of Example 2

\begin{tabular}{|l|l|l|}
\hline \multirow{2}{*}{$\boldsymbol{t}$} & \multicolumn{2}{|c|}{$\boldsymbol{m}=\mathbf{1 0 2 5}$} \\
\cline { 2 - 3 } & Exact & Approximation \\
\hline 0 & 1 & 1.001 \\
\hline 0.3 & 1.251 & 1.254 \\
\hline 0.6 & 1.39 & 1.391 \\
\hline 0.9 & 1.405 & 1.406 \\
\hline 1.2 & 1.294 & 1.295 \\
\hline 1.5 & 1.068 & 1.068 \\
\hline
\end{tabular}

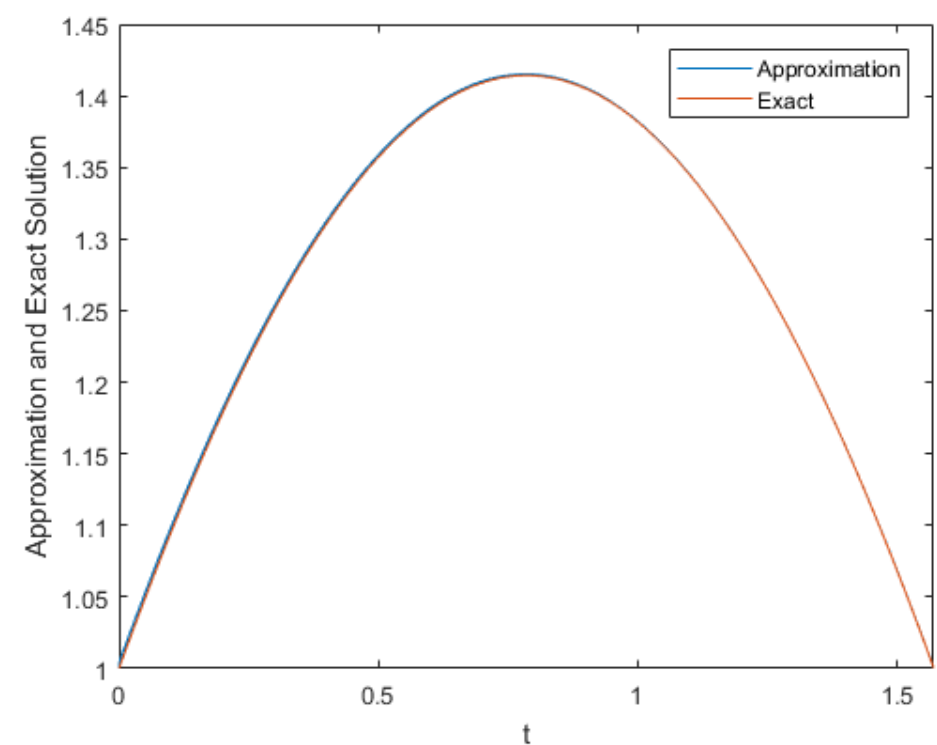

Figure 2: The Trajectory of The Exact and Approximation Solution of Example 2

Based on the graph above, it can be seen that the approximate and exact results coincide with each other at the interval $\left[0, \frac{\pi}{2}\right)$. At the beginning and end that if the number of partitions we take is getting bigger and $\varepsilon$ getting smaller, it will produce an approximation result that is closer to the exact.

\section{Conclusions}

The modified block pulse functions have better results by taking the partition size $(m)$ and $\varepsilon=\frac{1}{a}$ (provided $a>m$ so that $\frac{1}{a}<h$ ) than block pulse functions. The modified block pulse functions and their integration operational matrix are used to obtain the solution of mixed linear Volterra-Fredholm integral equations. Its applicability and accuracy of the proposed method was checked on some numerical examples. The results show that the value at any point of approximate and exact solutions has very little margin. We also illustrate both results to demonstrate of the proposed method have a good of efficiency and accuracy.

\section{References}

[1] Al-Saif, Nahdh S. M. and Ameen Sh. Ameen, 'Solving Mixed Volterra-Fredholm Integral Equation (MVFIE) by Designing Neural Network', Baghdad Science Journal, vol.16, pp. 116-120, 2019. 
[2] Chen, Zhong and Wei Jiang, 'An Approximate Solution for a Mixed Linear VolterraFredholm Integral Equation', Applied Mathematics Letters, vol.25, pp. 1131-1134, 2012.

[3] Deb, Anish and Srimanti Roychoudhury, Control System Analysis and Identification with MATLAB: Block Pulse and Related Orthogonal Functions. New York: CRC Press, 2019.

[4] Ghasemi, M., M. Fardi and R. Khoshsiar Ghaziani, 'Solution of System of the Mixed Volterra-Fredholm Integral Equations by An Analytical Method', Mathematical and Computer Modelling, vol.58, pp. 1522-1530, 2013.

[5] Hasan, Pakhshan M. A. and Nejmaddin Abdulla S., 'Numerical Solution of Mixed Volterra-Fredholm Integral Equations Using Linear Programming Problem', Applied Mathematics, vol.8, pp. 42-45, 2018.

[6] Maleknejad, K., M. Khodabin, and F. Hosseini Shekarabi, 'Modified Block Pulse Functions for Numerical Solution of Stochastic Volterra Integral Equations', Journal of Applied Mathematics, vol.4, pp. 1-10, 2014.

[7] Nemati, S., 'Numerical Solution of Volterra-Fredholm Integral Equations Using Legendre Collocation Method', Journal of Computational and Applied Mathematics, vol.278, pp. 29-36, 2015.

[8] Shahooth, Mohammed Khalid, 'Numerical Solution for Mixed Volterra-Fredholm Integral Equations of The Second Kind by Using Bernstein Polynomials Method', Mathematical Theory and Modeling, vol.5, pp. 154-162, 2015.

[9] Wazwaz, Abdul-Majid, A First Course in Integral Equations (Second Edition), Singapore: World Scientific, 2015.

[10] Wazwaz, Abdul-Majid, Linear and Nonlinear Integral Equations (Methods and Applications), Beijing and New York: Higher Education Press and Springer-Verlag Berlin Heidelberg, 2011. 